\title{
Intuitionistic Existential Instantiation and Epsilon Symbol
}

\author{
Grigori Mints
}

November 20, 2018

\begin{abstract}
A natural deduction system for intuitionistic predicate logic with existential instantiation rule presented here uses Hilbert's $\epsilon$-symbol. It is conservative over intuitionistic predicate logic. We provide a completeness proof for a suitable Kripke semantics, sketch an approach to a normalization proof, survey related work and state some open problems. Our system extends intuitionistic systems with $\epsilon$-symbol due to A. Dragalin and Sh. Maehara.
\end{abstract}

\section{Introduction}

In natural deduction formulations of classical and intuitionistic logic existenceelimination rule is usually taken in the form

$$
\begin{gathered}
A(a) \\
\frac{\vdots}{\exists^{\prime} A(x)} \quad{ }^{-}
\end{gathered}
$$

where $a$ is a fresh variable Existential instantiation is a rule

$$
\frac{\exists x A(x)}{A(a)} \exists i
$$

where $a$ is a fresh constant. It is sound and complete (with suitable restrictions) in the role of existence-elimination rule in classical predicate logic but is not sound intuitionistically, since it makes possible for example the following derivation:

$$
\begin{gathered}
\frac{C \rightarrow \exists x A(x), C \Rightarrow \exists x A(x)}{C \rightarrow \exists x A(x), C \Rightarrow A(a)} \\
\frac{\frac{C \rightarrow \exists x A(x) \Rightarrow C \rightarrow A(a)}{C \rightarrow \exists x A(x) \Rightarrow \exists x(C \rightarrow A(x))}}{\Rightarrow(C \rightarrow \exists x A(x)) \rightarrow \exists x(C \rightarrow A(x))}
\end{gathered}
$$


There are several approaches in the literature to introduction of restrictions making this rule conservative over intuitionistic predicate calculus.

We present an approach using intuitionistic version of Hilbert's epsilonsymbol and strengthening works by A. Dragalin [1] and Sh. Maehara [4] where $\epsilon$-terms are treated as partially defined. Then a survey of extensions and related approaches including important paper by K. Shirai [12] is given and some problems are stated.

We do not include equality since in this case adding of $\epsilon$-symbol with natural axioms is not conservative over intutionistic logic ([5, 11]). A simple counterexample due (in other terms) to C. Smorynsky [13] is

$$
\forall x \exists y P(x, y) \rightarrow \forall x x^{\prime} \exists y y^{\prime}\left(P x y \& P x^{\prime} y^{\prime} \&\left(x=x^{\prime} \rightarrow y=y^{\prime}\right)\right)
$$

In our natural deduction system NJ $\epsilon$ axioms and propositional inference rules are the same as in ordinary intuitionistic natural deduction, the same holds for $\forall$-introduction. The remaining rules are as follows:

$$
\frac{\Gamma \Rightarrow \exists x F(x)}{\Gamma \Rightarrow F(\epsilon x F(x))} \exists i
$$

existential instantiation,

$$
\frac{\Gamma \Rightarrow t \downarrow \quad \Delta \Rightarrow \forall z F(z)}{\Gamma, \Delta \Rightarrow F(t)} \quad \frac{\Gamma \Rightarrow t \downarrow \quad \Delta \rightarrow F(t)}{\Gamma, \Delta \Rightarrow \exists z F(z)}
$$

where

$$
\epsilon x A(x) \downarrow:=\exists y(\exists x A(x) \rightarrow A(y)),
$$

and $t \downarrow:=\top$ (the constant "true") if $t$ is a variable or constant.

Two semantics are given for $\mathrm{NJ} \epsilon$, or more precisely to an equivalent Gentzenstyle system IPC $\epsilon$ (Section 2). The first semantics, which is incomplete but convenient for a proof of conservative extension property over IPC is defined in Section 3 . 4.

The second semantics with a completeness proof for IPC $\epsilon$ is given in Section

Section 5 presents a sketch of a possible proof of a normal form theorem. Section 6 surveys some of the previous work and Section 17 outlines some open problems.

\section{Gentzen-style system IPC $\epsilon$}

Let us state our Gentzen-style rules for the intuitionistic predicate calculus IPC $\epsilon$ with $\epsilon$-symbol. For simplicity we assume that the language does not have function symbols except constants. Formulas and terms are defined by familiar inductive definition plus additional clause:

If $A(x)$ is a formula then $\epsilon x A(x)$ is a term. 
Derivable objects of IPC $\epsilon$ are sequents $\Gamma \Rightarrow A$ where $\Gamma$ is a finite set of formulas, $A$ is a formula. This means in particular that structural rules are implicitly included below.

First, let's list the rules of the intuitionistic predicate calculus IPC without $\epsilon$-symbol.

Axioms:

$$
\Gamma, A \Rightarrow A, \quad \Gamma, \perp \Rightarrow A
$$

Inference rules:

$$
\begin{gathered}
\frac{\Gamma \Rightarrow A \quad \Gamma \Rightarrow B}{\Gamma \Rightarrow A \& B} \Rightarrow \& \quad \frac{A, B, \Gamma \Rightarrow G}{A \& B, \Gamma \Rightarrow G} \& \Rightarrow \\
\frac{A, \Gamma \Rightarrow G \quad B, \Gamma \Rightarrow G}{A \vee B, \Gamma \Rightarrow G} \vee \Rightarrow \quad \frac{\Gamma \Rightarrow A}{\Gamma \Rightarrow A \vee B} \Rightarrow \vee \frac{\Gamma \Rightarrow B}{\Gamma \Rightarrow A \vee B} \\
\frac{\Gamma \Rightarrow A \quad B, \Gamma \Rightarrow G}{A \rightarrow B, \Gamma \Rightarrow G} \rightarrow \Rightarrow \quad \frac{A, \Gamma \Rightarrow B}{\Gamma \Rightarrow A \rightarrow B} \Rightarrow \rightarrow \\
\frac{\Gamma \Rightarrow A(t)}{\Gamma \Rightarrow \exists x A(x)} \Rightarrow \exists \quad \frac{\Gamma \Rightarrow A(b)}{\Gamma \Rightarrow \forall x A(x)} \Rightarrow \forall \\
\frac{A(b), \Gamma \rightarrow G}{\exists x A(x), \Gamma \Rightarrow G} \exists \quad \frac{A(t), \Gamma \rightarrow G}{\forall x A(x), \Gamma \Rightarrow G} \forall \Rightarrow \\
\frac{\Gamma \rightarrow C \quad C, \Gamma \Rightarrow G}{\Gamma \rightarrow G} C u t
\end{gathered}
$$

For IPC $\epsilon$ quantifier-inferences $\Rightarrow \exists, \forall \Rightarrow$ are modified by requirement that the term $t$ substituted in the rule should be "defined" (cf. (3) ).

$$
\frac{\Gamma \Rightarrow t \downarrow \quad F(t), \Delta \Rightarrow \Delta}{\forall z F(z), \Gamma, \Delta \Rightarrow \Delta} \forall \Rightarrow \quad \frac{\Gamma \Rightarrow \Delta, t \downarrow \quad \Gamma \rightarrow \Delta, F(t)}{\Gamma \Rightarrow \Delta, \exists z F(z)} \Rightarrow \exists
$$

$\exists \Rightarrow$-rule is also changed for IPC $\epsilon$ :

$$
\frac{A(\epsilon x A(x)) \Gamma \Rightarrow G}{\Gamma, \exists x A(x) \Rightarrow G} \exists_{\epsilon} \Rightarrow
$$

A routine proof shows that IPC $\epsilon$ is equivalent to a Hilbert-style system obtained by weakening familiar axioms for quantifiers to

$$
\begin{aligned}
& (\epsilon Q 1) t \downarrow \& \forall x A(x) \rightarrow A(t) \\
& (\epsilon Q 2) t \downarrow \& A(t) \rightarrow \exists x A(x)
\end{aligned}
$$

and adding the axiom

$$
\exists x A(x) \rightarrow A(\epsilon x A(x))
$$




\subsection{Equivalence of IPC $\epsilon$ and $\mathrm{NJ} \epsilon$}

Let us remind that in natural deduction a sequent

$$
A_{1}, \ldots, A_{n} \Rightarrow A
$$

is used to indicate that $\mathrm{A}$ is deducible from assumptions $A_{1}, \ldots, A_{n}$.

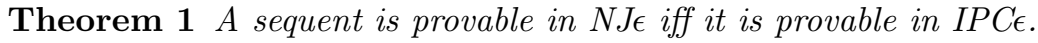

Proof. The proof is routine: every rule of one of these systems is directly derivable in the other system. Let's show derivations of the rules $\exists i$ and $\exists \Rightarrow$ from each other using abbreviation $e:=\epsilon x F(x)$.

$$
\frac{\Gamma \rightarrow \exists x F(x) \quad \frac{F(e) \Rightarrow F(e)}{\exists x F(x) \Rightarrow F(e)}}{\Gamma \Rightarrow F(e)} \exists \Rightarrow \quad \frac{\exists x F(x) \Rightarrow \exists x F(x)}{\exists x F(x) \Rightarrow F(e)} \exists i \frac{F(e), \Gamma \Rightarrow G}{\Gamma \Rightarrow F(e) \rightarrow G}
$$

\section{A Kripke Semantics for Intuitionistic $\epsilon$-symbol}

To prove that IPC $\epsilon$ is conservative over IPC we present an incomplete semantics modifying a semantics from [1]. The main modification is in the definition of $t \downarrow$ and treatment of atomic formulas containing $\epsilon$-terms $\epsilon x A$.

Definition 1 Let $w$ be a world in a Kripke model. Denote

$$
\epsilon x A(x) \downarrow w: \equiv w \models \epsilon x A(x) \downarrow .
$$

We say that a term $\epsilon x A$ is defined in $w$ iff $\epsilon x A(x) \downarrow w$.

Symbol $\perp$ in in next definition indicates the condition (6) below.

Definition 2 An intuitionistic Kripke $\epsilon \perp$-model (or simply model in this section)

$$
\mathcal{M}=(W,<, D, \models, V)
$$

has to satisfy the following conditions:

$(W,<)$ is a Kripke frame with a strict partial ordering $<$,

$D$ is a domain function assigning to every $w \in W$ a non-empty set $D(w)$ monotone with respect to $<$,

$w \models A$ is a relation between worlds $w \in W$ and atomic formulas $A$ with constants from

$$
D:=\cup_{w \in W} D(w)
$$

monotonic with respect to $\leq$ and such that

$$
w \not \models A \text { if } A \text { contains at least one constant in } D-D(w) \text {. }
$$

$V$ is a valuation function assigning a constant $V(e, w) \in D$ to any $\epsilon$-term $e$ (possibly containing constants from $D$ ) and $w \in W$. 
The relation $\models$ is extended to composite formulas in the familiar way. The components of an $\epsilon$-model have to satisfy following conditions.

$$
\begin{gathered}
V(\epsilon x B(x, \epsilon y C), w)=V(\epsilon x B(x, V(\epsilon y C, w)), w), \\
w \models A(\epsilon y C) \leftrightarrow w \models A(x, V(\epsilon y C)),
\end{gathered}
$$

where substitution of $\epsilon y C$ is safe, that is no free variable of $\epsilon y C$ becomes bound. Also

if $e \downarrow w$ for a term $e:=\epsilon x A(x)$, then

$$
V(e, w) \in D(w) \text { and } V\left(e, w^{\prime}\right)=V(e, w) \text { for every } w^{\prime} \geq w .
$$

Note once more that an atomic formula $P\left(d_{1}, \ldots, d_{n}\right)$ is false in a world $w$ if at least one of $d_{i}$ is not in $D(w)$.

This leads to incompleteness, for example formula

$$
P(\epsilon x P(x)) \rightarrow \exists x P(x)
$$

is valid: if $\epsilon x P(x)$ is undefined in a world $w$ then the premise is false in $w$, otherwise the conclusion is true. However this formula is not derivable, since it implies $(C \rightarrow \exists x P(x)) \rightarrow \exists x(C \rightarrow P(x))$.

The proofs of the next lemmata are routine.

Lemma 1 Let $t$ be a closed term, A a closed formula with constants from D. Then

$$
w \leq w^{\prime} \rightarrow\left(t \downarrow w \rightarrow t \downarrow v^{\prime} \&\left(w \models A \rightarrow w^{\prime} \models A\right)\right)
$$

Proof. Simultaneous induction on $t, A$.

Lemma 2 If $\Gamma$ is a set of formulas, $G$ a formula then $\Gamma \vdash G$ in IPC $\epsilon$ implies $\Gamma \models G$.

Proof. Induction on derivations. Checking the rule $\exists \Rightarrow$ uses the fact that $\exists x A(x)$ implies $\epsilon x A(x) \downarrow$. It may be interesting to check whether any other properties of the formula $t \downarrow$ are used.

Theorem 2 If $A, B$ formulas without $\epsilon$-symbol then $A \vdash B$ in IPC $\epsilon$ implies $A \vdash B$ in intuitionistic predicate logic IPC.

Proof. We need to prove that for every Kripke model

$$
\mathcal{M}_{0}=\left(W,<, D, \models_{0}\right)
$$

for intutionistic predicate logic refuting $A \rightarrow B$ there is an IPC $\epsilon$-model refuting $A \rightarrow B$. Before applying the construction from [1], let us recall a refinement of a completeness theorem for intuitionistic predicate logic IPC.

Lemma 3 The following additional requirements to the definition of Kripke frame $(W,<, D)$ for IPC are still complete: 
1. $W$ is a countable tree with a root $\mathbf{0}$ such that each $w \in W$ except $\mathbf{0}$ has unique immediate <-predecessor and the number of predecessors of $w$ is finite.

2. domains $D(w)$ are strictly increasing: if $w<w^{\prime}$ then $D(w)$ is a proper subset of $D\left(w^{\prime}\right)$.

Proof. The requirement 1 is satisfied by the canonical proof search tree for a given sequent, see for example [8]. To satisfy the second requirement, note that an infinite branch of the canonical proof search tree does not have "leaf worlds": for every $w \in W$ there exists a $w^{\prime}>w$. Now take a fixed element $e \in D\left(w_{0}\right)$ and duplicate it by a fresh element, say $e_{w}$ in every world $w$. More precisely for the new domain function $D^{\prime}$ define

$$
e_{w} \in D^{\prime}(w)-D^{\prime}\left(w^{-}\right),
$$

where $w^{-}$is the immediate predecessor of $w$. Let's extend the relation $\models$ by identifying $e_{w}$ and $e$, more precisely define for atomic formulas $P\left(c_{1}, \ldots, c_{n}\right)$ with constants $c_{i} \in D^{\prime}(w)$

$$
w \models P\left(c_{1}, \ldots, c_{n}\right):=w \models P\left(c_{1}^{-}, \ldots, c_{n}^{-}\right)
$$

where $c_{i}^{-}=e$, if $c_{i}=e_{w}$ and $c_{i}^{-}=c_{i}$ otherwise. It is easily proved by induction on formulas that this property extends to all formulas:

$$
w \models A\left(c_{1}, \ldots, c_{n}\right) \text { implies } w \models A\left(c_{1}^{-}, \ldots, c_{n}^{-}\right)
$$

so that the new model verifies (and refutes) the same formulas. $\dashv$

Proof of the Theorem 2. We extend the model for IPC satisfying the previous Lemma by the definition of values for $\epsilon$-terms without changing domains $D(w)$, which is done by induction on construction of the term. Assume that the elements of $D$ are well-ordered by a relation $\prec$ in some arbitrary way. In view of the condition (77) it enough to define $V(\epsilon x A, w)$ when $\epsilon x A$ does not have proper non-closed $\epsilon$-subterms. In that case,

if $\epsilon x A(x) \downarrow w$, take the <-minimal element $v \leq w$ such that $\epsilon x A \downarrow v$, then define

$$
V(\epsilon x A(x), w):=\text { the } \prec \text {-first } d \in D(v)(v \models(\exists x A(x) \rightarrow A(d)))
$$

If not $\epsilon x A(x) \downarrow w$, define $V(\epsilon x A(x), w)$ as the $\prec$-first $d \in D-D(w)$.

\section{Completeness proof for IPC $\epsilon$}

We prove that removing condition (6) but preserving familiar monotonicity requirement

$$
w \leq w^{\prime} \rightarrow\left(w \models A \rightarrow w^{\prime} \models A\right)
$$

leads to a complete semantics for IPC $\epsilon$.

For simplicity consider term models where individual domain $D(w)$ for every world $w$ consists of terms, and evaluation function for terms is identity: value of a term $t$ is $t$. In particular the value of $\epsilon x A$ is $\epsilon x A$. 
Definition 3 An intuitionistic Kripke (term) $\epsilon$-model (or simply $\epsilon$-model)

$$
\mathcal{M}=(W,<, D, \models, V)
$$

has to satisfy the following conditions.

$(W,<)$ is a Kripke frame with a strict partial ordering $<$,

$D$ is a domain function assigning to every $w \in W$ a non-empty set $D(w)$ (of terms) monotone with respect to $<$, from

$w \models A$ is a relation between worlds $w$ and atomic formulas $A$ with constants

$$
D:=\cup_{w \in W} D(w)
$$

monotonic with respect to $\leq$.

$V$ is a valuation function assigning a constant $V(e, w) \in D$ to any $\epsilon$-term $e$ (possibly containing constants from $D)$ and $w \in W$. (In a term model $V(e, w)=$ e).

The relation $\models$ is extended to composite formulas in the familiar way. The components of an $\epsilon$-model have to satisfy following conditions.

$$
\begin{gathered}
V(\epsilon x B(x, \epsilon y C), w)=V(\epsilon x B(x, V(\epsilon y C, w)), w) \\
w \models A(\epsilon y C) \leftrightarrow w \models A(x, V(\epsilon y C))
\end{gathered}
$$

where substitution of $\epsilon y C$ is safe, that is no free variable of $\epsilon y C$ becomes bound. Also if $e \downarrow w$ for a term $e:=\epsilon x A(x)$, then

$$
V(e, w) \in D(w) \text { and } V\left(e, w^{\prime}\right)=V(e, w) \text { for every } w^{\prime} \geq w .
$$

Let's present a completeness proof along familiar lines.

Definition 4 An infinite sequent is a pair of sets $\Gamma, \Delta$ of formulas such that there is an infinite number of variables not in $\Gamma \cup \Delta$. An infinite sequent $w$ is written as $\Gamma \Rightarrow \Delta$ and notation

$$
w_{a}:=\Gamma, w_{s}:=\Delta
$$

is used for its antecedent and succedent.

$L_{w}$ denotes the set of all terms and formulas with free variables and constants occurring in formulas of $w$.

$D(w)$ is the set of all terms $t \in L_{w}$ such that $(t \downarrow) \in w_{a}$. In other worlds $D(w)$ consists of all free variables and constants in $w$ plus all $\epsilon$-terms $\epsilon x A(x)$ such that $\exists y(\exists x A(x) \rightarrow A(y)) \in w_{a}$.

An infinite sequent $w$ is consistent, if it is underivable, that is if no finite sequent $\Gamma \Rightarrow \Delta$ with $\Gamma \subset w_{a}, \Delta \subset w_{s}$ is derivable in IPC $\epsilon$.

$A$ consistent infinite sequent $w$ is maximal consistent if $w_{a} \cup w_{s}$ is the whole set of formulas in $L_{w}$.

Lemma 4 Every consistent infinite sequent $w_{0}$ can be extended to a maximal consistent sequent. 
Proof. Enumerate all formulas containing only free variables and constants in $L_{w_{0}}$, then add them one by one to $w_{a}$ or $w_{s}$ preserving consistency. At the $n$-th stage of this process a sequent $w_{n}$, an extension of $w_{0}$ by a finite number of formulas is generated.

It cannot happen that at some stage $n$ of this process a formula $A$ fits none of $w_{a}^{n}, w_{s}^{n}$, i.e., both of

$$
w_{a}^{n} \Rightarrow w_{s}^{n}, A_{n} \quad A_{n}, w_{a}^{n} \Rightarrow w_{s}^{n}
$$

are inconsistent, since in that case $w_{a}^{n} \Rightarrow w_{s}^{n}$ is inconsistent by a cut rule.

Important example. If $w$ is $\forall x P(x) \Rightarrow P(\epsilon x Q(x))$ with $P \neq Q$, and the first "undecided" formula is $\exists y(\exists x Q(x) \rightarrow Q(y))$ then this formula is added to the succedent, since adding it to the antecedent results in an inconsistent sequent.

Lemma 5 Every maximal consistent infinite sequent $w$ is closed under invertible rules of multiple-succedent version of IPC $\epsilon$, that is under all rules except $\Rightarrow \forall, \Rightarrow \rightarrow$. More precisely

$$
\begin{gathered}
(A \& B) \in w_{a} \text { implies } A \in w_{a} \text { and } B \in w_{a}, \\
(A \rightarrow B) \in w_{a} \text { implies } A \in w_{s} \text { or } B \in w_{a}, \\
(A \vee B) \in w_{a} \text { implies } A \in w_{a} \text { or } B \in w_{a}, \\
(\forall x A(x)) \in w_{a} \text { implies }(\forall t \in D(w))\left(A(t) \in w_{a}\right) \\
(\exists x A(x)) \in w_{a} \text { implies } A(\epsilon x A(x)) \in w_{a} \\
(A \vee B) \in w_{s} \text { implies } A \in w_{a} \text { and } B \in w_{a}, \\
(A \& B) \in w_{s} \text { implies } A \in w_{a} \text { or } B \in w_{a}, \\
(\exists x A(x)) \in w_{s} \text { implies }(\forall t \in D(w))\left(A(t) \in w_{s}\right)
\end{gathered}
$$

Proof. Suppose $(A \& B) \in w_{a}$. If $A \notin w_{a}$ then by maximality $A \in w_{s}$. Therefore $w$ is inconsistent.

Suppose $\forall x A \in w_{a}$. If $A(t) \notin w_{a}$ for some $t \in D(w)$ then by maximality $A(t) \in w_{s}$. Therefore $\forall x A \Rightarrow A(t)$ is derived by one application of the $\forall \Rightarrow$ rule, and hence $w$ is inconsistent. Note that additional premise $t \downarrow$ of this rule is available by $t \in D(w)$.

Other cases are similar.

Definition 5 For infinite sequents $w, w^{\prime}$ define

$$
w<w^{\prime} \text { iff } w_{a} \subseteq w_{a}^{\prime} \text { and } D(w) \subseteq D\left(w^{\prime}\right)
$$

Lemma 6 The set of maximal consistent sequents is closed under non-invertible rules $\Rightarrow \rightarrow, \Rightarrow \forall$. More precisely,

For every maximal consistent sequent $w$, if $(A \rightarrow B) \in w_{s}$ then there exists a maximal consistent sequent $w^{\prime}>w$ with $A \in w_{a}^{\prime}, B \in w_{s}^{\prime}$.

For every maximal consistent sequent $w$, if $\forall x A(x) \in w_{s}$ then there exists a maximal consistent sequent $w^{\prime}>w$ with $A(a) \in w_{s}^{\prime}$ for some variable $a$, $a \in D\left(w^{\prime}\right)$. 
Proof. If $(A \rightarrow B) \in w_{s}$ then the sequent $A, w_{a} \Rightarrow B$ is consistent, since otherwise one application of the rule $\Rightarrow \rightarrow$ leads to inconsistency of $w$. Now extend $A, w_{a} \Rightarrow B$ to a complete consistent sequent.

If $\forall x A(x) \in w_{s}$ then the sequent $w_{a} \Rightarrow A(a)$ for a fresh variable $a$ is consistent, since otherwise one application of the rule $\Rightarrow \forall$ leads to inconsistency of $w$. Now extend $A, w_{a} \Rightarrow B$ to a complete consistent sequent.

Definition 6 (Canonical model) Consider the following model

$$
M=(W,<, V, \models) .
$$

$W$ is the set of all maximal complete sequents, $<, V$ are as above, $w \models A$ iff $A \in w_{a}$ for atomic formulas $A$.

This definition implies that $w \not \models A$ for atomic $A \in w_{s}$, since otherwise $w$ is inconsistent.

Lemma 7 The relation $\models$ for atomic formulas and the function $D$ is monotonic.

Proof. Consider only $D(w)$. Let $w<w^{\prime}$. All variables and constants in $D(w)$ are in $D\left(w^{\prime}\right)$ by the definition of $<$. Assume $\epsilon x A(x) \in D(w)$, that is $\epsilon x A(x) \downarrow \in w_{a}$. Then $\epsilon x A(x) \downarrow \in w_{a}^{\prime}$ by $w<w^{\prime}$, and hence $\epsilon x A(x) \in D\left(w^{\prime}\right)$. $\dashv$

Lemma 8 For every formula $A \in L_{w}$

1. A $\in w_{a}$ implies $w \models A$,

2. $A \in w_{s}$ implies $w \not \models A$,

Proof. Induction on formulas using Lemmata 516. For example, if $A \& B \in w_{a}$ then $A, B \in w_{a}$, therefore $w \models A, w \models B$ by induction hypothesis, and hence $w \models A \& B$.

If $\forall x A \in w_{s}$ then there exists $w^{\prime}>w$ such that $A(a) \in w_{s}^{\prime}$ for some variable $a \in D\left(w^{\prime}\right)$. Therefore $w^{\prime} \not \forall A(a)$ and hence $w \not \forall \forall x A(x)$.

Theorem 3 The system IPCE is sound and complete.

Proof. Soundness is checked as before. For completeness take arbitrary underivable formula $A$, then extend sequent $\Rightarrow A$ to a maximal consistent set $w$. By previous Lemma $w \not \models A$.

\section{Cut-free Formulation, Normal Natural Deduc- tion}

It is plausible that completeness proof for the rules with cut given in a previous section 4 can be modified to provide completeness of a cut-free formulation. As our examples above show, complete cut-elimination is impossible. One has 
to admit cuts for formulas of the form $\epsilon x A(x) \downarrow$ where $\epsilon x A(x)$ occurs in the conclusion. The following proof where $e:=\epsilon x P(x)$ is an example.

$$
\begin{aligned}
& \frac{\exists x P(x) \Rightarrow e \downarrow \quad \neg P(e), P(e), \exists x P(x) \Rightarrow P(0)}{\frac{\forall x \neg P(x), P(e), \exists x P(x) \Rightarrow P(0)}{\forall x \neg P(x), P(e) \Rightarrow \exists x P(x) \rightarrow P(0)}} \\
& \frac{\frac{\forall x \neg P(x), P(e) \Rightarrow \exists y(\exists x P(x) \rightarrow P(y))}{\forall x \neg P(x), P(e) \Rightarrow}}{\exists y(\exists x P(x) \rightarrow P(y)) \Rightarrow e \downarrow \quad \neg P(e), P(e) \Rightarrow}
\end{aligned}
$$

Let's outline a possible proof that this restriction is complete.

First, the definition of the canonical model should be modified along the lines of a proof by M. Fitting [2] (cf. also [8]). Our definition of a complete consistent sequent in the section 4 requires that such a sequent $w$ contains every formula of its language $L_{w}$ as a member of its antecedent or succedent. This requirement is weakened as follows.

For any formula $F$ in $L_{w}$ either $F \in w_{a} \cup w_{b}$ or there is a clash: both sequents

$$
w_{a} \Rightarrow w_{b}, F \text { and } F, w_{a} \Rightarrow w_{b}
$$

are cut-free derivable. This should provide completeness of a multiple-sequent cut-free formulation. Then equivalence to a cut-free one-succedent formulation should be proved by pruning and permutation of inferences as in 8 . Finally cutfree one-succedent derivations are transformed into a normal natural deductions as in [7.

\section{Comparison with Previous Work}

\subsection{System $I P C \Omega \epsilon$}

Let $\exists \epsilon x A(x):=\exists x A(x)$.

A. Dragalin's system $I P C \Omega \epsilon$ from [1] for a given language $\Omega \epsilon$ is obtained by weakening familiar axioms for quantifiers

$$
\begin{aligned}
& (\epsilon Q 1) \exists t \& \forall x A(x) \rightarrow A(t) \\
& (\epsilon Q 2) \exists t \& A(t) \rightarrow \exists x A(x)
\end{aligned}
$$

and adding the axiom

$$
\exists x A(x) \rightarrow A(\epsilon x A(x))
$$

A. Dragalin in [1] tried to avoid as much as possible dealing with a value of an $\epsilon$-term in a world $w$ where the term is not defined. Values (in a given world $w)$ are assigned only to $\epsilon$-terms defined in $w$, and many intermediate results are proved only for the case when all relevant $\epsilon$-terms are defined. Nevertheless soundness is established for all formulas, without any restrictions. As pointed earlier, this system is not complete.

In Section 3 we changed the definition of a model from 1 to a more uniform version: $\epsilon$-term $e$ which is not defined at the world $w$ is assigned a value at 
$w$, but this value does not belong to the individual domain $D(w)$. To make this possible, the Kripke frame underlying the model and the domain function should satisfy additional conditions that still guarantee completeness.

Let us consider other systems in the literature.

\subsection{Systems with $\exists y(\exists x A(x) \rightarrow A(y)$ as Existence Condition}

In systems due to to Sh. Maehara and K. Shirai 4, 12, instead of using $\exists x A(x)$ as a discriminating criterion, a weaker formula $\exists y(\exists x A(x) \rightarrow A(y))$ is employed. This still allows to anticipate a correct future value of the term $\epsilon x A(x)$ in a world $w$ even if $\exists x A(x)$ fails in $w$.

Sh. Maehara treats weaker language than ours: $\epsilon x A(x)$ is a syntactically correct term only if it is closed. He proves (using partial cut-elimination and other syntactic transformations) conservativity over IPC of the rules

$$
\begin{gathered}
\frac{\Gamma, \exists x A(x) \quad A(\epsilon x A(x)), \Delta \Rightarrow G}{\Gamma, \Delta \Rightarrow G} \exists_{\epsilon} \\
\frac{\Gamma \Rightarrow t \downarrow \quad F(t), \Delta \Rightarrow G}{\forall z F(z), \Gamma, \Delta \Rightarrow G} \quad \frac{\Gamma \Rightarrow t \downarrow \quad \Delta \rightarrow F(t)}{\Gamma, \Delta \Rightarrow \exists z F(z)}
\end{gathered}
$$

where

$$
\epsilon x A(x) \downarrow:=\exists y(\exists x A(x) \rightarrow A(y)) ; a \downarrow:=\top
$$

Here $T$ is the constant true, $a$ is an arbitrary variable.

Note that the first of these rules contains a hidden cut. This conservativity result is used to establish a kind of completeness theorem for IPC over a modification of Kripke semantics, although this modification is not stated explicitly. More precisely, Sh. Maehara proves Kripke-style soundness and completeness result for the relation $A \in \alpha$ between formulas $A$ and complete consistent (in his sense) subsets $\alpha$ of the set of formulas. Only his condition for $\forall$ is not standard:

$\forall x A(x) \in \alpha \leftrightarrow(\exists B)\left(B \in \alpha \& \forall \beta \forall t\left[B \in \beta \rightarrow\left(t \in D_{\beta} \rightarrow A(t) \in \beta\right)\right]\right)$

To establish this condition he uses admissibility of the following rule in his system:

$$
\frac{\exists y(\exists x \neg A(x) \rightarrow \neg A(y)) \rightarrow A(\epsilon x \neg A(x))}{\forall x A(x)}
$$

This rules approximates equivalence

$$
\forall x A(x) \leftrightarrow A(\epsilon x \neg A(x))
$$

which is valid only classically.

K. Shirai [12] removes restriction to closed $\epsilon$-terms. He considers a language with the existence predicate denote by $D$. Instead of rules used by Maehara he considers following axioms:

$$
\begin{gathered}
D(t), \exists y(\exists x A(x, t) \rightarrow A(y, t) \Rightarrow D(\epsilon x A(x)) \\
D(t), \exists x A(x, t) \Rightarrow A(\epsilon x A(x, t), t)
\end{gathered}
$$


plus standard modifications of quantifier rules for the system with existence predicate D.

He proves conservativity of his system over IPC by a combination of a partial cut-elimination and Maehara's argument.

D. Leivant 3 and V. Smirnov [14 define logical systems with $\epsilon$-symbol conservative over IPC by requiring that assumptions discharged in natural deduction rules contain no $\epsilon$-symbol. These systems are probably much weaker than IPC $\epsilon$. The system introduced by the author in [9] is certainly weaker than IPC $\epsilon$ : a sequent containing subterm $\epsilon x A(x, y)$ with a bound variable $y$ is syntactically correct only provided $\forall y \exists x A(x, y)$ is a member of the antecedent.

\section{Further Work}

Complete proof of cut-elimination for IPC $\epsilon$ and of normal form theorem for NJ $\epsilon$.

Give a syntactic proof of cut-elimination for IPC $\epsilon$ and of normalization for NJ $\epsilon$.

Provide a semantics for the systems by Sh. Maehara and K. Shirai [4, 12 ] and find out whether these systems admit cut-elimination. It seems that the system by Shirai provides the most general formulation of the idea that $\epsilon$-terms is partially defined in some arbitrary way. The restriction $D(t)$ allowing to quantify over value of $t$ can be arbitrary predicate with the only condition (14).

\section{References}

[1] Dragalin, A. Intuitionistic Logic and Hilbert's $\epsilon$-symbol, (Russian) Istoriia i Metodologiia Estestvennykh Nauk, Moscow, MGU, 1974, s. 78-84, republished in: Albert Grigorevich Dragalin, Konstruktivnaia Teoriia Dokazatelstv I Nestandartnyi Analiz, s. 255-263, Moscow, Editorial Publ. )

[2] Fitting, M., Intuitionistic Logic, Model Theory And Forcing, Amsterdam, North-Holland, 1969

[3] Leivant, D., Existential instantiation in a system of natural deduction for intuitionistic arithmetics, Technical Report ZW 13/73, Stichtung Mathematisch Centrum, Amsterdam, 1973

[4] Maehara, Sh., A General Theory of Completeness Proofs, Ann. Jap. Assoc. Phil. Sci., 1970, no. 3, p. 242-256

[5] Mints, G., The Skolem Method in Intuitionistic Calculi. Proc. Inst. Steklov, 121, AMS, 1974, p. 73-109

[6] Mints, G., Skolem Method of Elimination of Positive Quantifiers in Sequential Calculi, Soviet Math. Dokl. 7, no.4, 1966, 861-864

[7] Mints, G., Linear Lambda-terms and Natural Deduction, Studia Logica, 60, 1998, p. 209-231 
[8] Mints, G., A Short Introduction to Intuitionistic Logic , Kluwer Academic/ Plenum Publishers, 2000

[9] Mints, G., Heyting Predicate Calculus with Epsilon Symbol (Russian), Zapiski Nauchnykh Seminarov Leningradskogo Otdeleniya Matematicheskogo Instituta im. V. A. Steklova AN SSSR, Vol. 40, pp. 110-118, 1974, English Translation in [10] p. $97-104$

[10] Mints, G., Selected Papers in Proof Theory, Bibliopolis/North-Holland, 1992

[11] Osswald, H., Über Skolemerweiterungen in der Intuitionistichen Logik mit Gleichheit, Lecture Notes in Mathematics, 1975, Volume 500, 1975, p. 264266 ,

[12] Shirai, K. Intuitionistic Predicate Calculus with $\epsilon$-symbol, 1971, no.4, p. 49-67

[13] Smorynski C., On Axiomatizing Fragments, J. Symbolic Logic, v. 42, no.4, 1977, p. $530-544$

[14] Smirnov, V., Theory of quantification and E-calculi. In: Essays on mathematical and philosophical logic, Essays on Mathematical and Philosophical Logic: Proceedings of the Fourth ... By Jaakko Hintikka, Ilkka Niiniluoto, Esa Saarinen, Proc. 4th Scand. Logic Symp., Kluwer, 1979, p. 41-49 